\title{
Gig Health vs eHealth: Future Prospects in Saudi Arabian Health-Care System
}

\section{Turki M Alanzi (D)}

Health Information Management and Technology Department, College of Public Health, Imam Abdulrahman Bin Faisal University, Dammam, Saudi Arabia
Introduction: The number of gig workers has been rising across the world, reflecting the growing trend toward the gig economy, which is already functioning in various sectors, such as transport(eg, Uber), technology (eg, Appen), and others (eg, Upwork). However, application of gig economy in health care is underresearched, especially in areas of eHealth that can facilitate various online activities and be outsourced.

Purpose: The purpose of this study was to investigate the gig economy and eHealth in terms of prospects for future health care in Saudi Arabia.

Methods: Analysis of data collected using online semistructured interviews with 19 experts, including executives from ministries of health and information and communications, physicians, and health-care administrative managers was conducted and new empirical insights are offered.

Results: A lack of legal and regulatory frameworks and integrating gig culture into the eHealth system were identified to be the major challenges. Improved operational efficiency of health care, reduced costs, and positive societal impact were identified to be the opportunities and benefits of using the gig economy and eHealth in Saudi Arabia. A strategic framework and an effective regulatory framework are essential for integrating the gig economy into the eHealth system in Saudi Arabia and improving future health care by adopting innovative artificial intelligence and machine-learning technologies.

Conclusion: eHealth has major scope for future health-care operations in Saudi Arabia and use of the gig economy in eHealth may lead to effective management of eHealth operations. More studies are required to evaluate the idea of integrating the gig economy and eHealth and analyze its impact.

Keywords: health care, gig economy, gig health, prospects, eHealth

\section{Introduction}

Health-care challenges across the world have been increasing in the last few years. According to the World Health Organization, chronic-disease prevalence is expected to rise by $57 \%$ by the year $2020 .{ }^{1}$ In addition, frequent pandemics, such as Ebola, SARS, MERS, and H1N1, have made the situation further challenging by increasing the burden on health care in terms of treating both regular patients and infected patients during outbreaks. Though the burden on health care is increasing, global health-care resources are inadequate to meet the demand for rising health-care needs. Globally, for every 10,000 population, only ten doctors are available, making access to health care further challenging. ${ }^{2}$ Not only a lack of sufficient health-care professionals but also issues in other areas of health care have also been observed. For example, a global health index score of 40.2 out of 100 reveals various issues, such as ineffective
Correspondence: Turki M Alanzi Health Information Management and Technology Department, College of Public Health, Imam Abdulrahman Bin Faisal University, Dammam, Saudi Arabia, Tel +966 13-333-1211

Email talanzi@iau.edu.sa 
response to health-care needs, poor services and quality, and poor deployment of health-care technologies. ${ }^{3}$ While some regions have good health-care resources, a few, such as Africa, have fewer than three doctors per 10,000 population and also facing many other health-care challenges. It has been observed that most developing countries face many challenges in meeting growing health-care needs and transforming traditional health-care systems to digital ones. ${ }^{3}$

Saudi Arabia is one of the fastest-developing countries in the Middle East and has been undergoing rapid transformations through Vision 2030 initiatives, which focus on moving toward a knowledge-based economy from an oildependent one. Access to quality health care is one of the major objectives of Vision 2030, as a result of which major initiatives in digitizing health-care operations have been launched. ${ }^{4}$ A steep increase in the health-care budget was initiated with the objective of modernizing health-care services and increasing the skilled health-care workforce, ${ }^{5,6}$ in order to reduce dependence on expatriate health- workers. ${ }^{7}$ However, according to a recent study, ${ }^{5}$ Saudi Arabia ranked 89th globally in prevention of diseases, 114th in responding to health-care needs, 81 in health norms and compliances, and 71st in severity of health-care risks among 195 countries, indicating challenges in various areas of health care. ${ }^{8}$ Moreover, the number of critical health conditions, such as diabetes and coronary diseases, have ben rapidly increasing in Saudi Arabia in the last few years, ${ }^{9,10}$ further increasing the health-care burden on an already-exhausted system due to the recent COVID-19 pandemic. With the rising healthcare needs, it is estimated that there is a need for an additional 13,700 doctors, $>30,000$ other health-care workers, and $>20,000$ beds in Saudi Arabia. ${ }^{11}$ Furthermore, an increasing trend of psychological distress, eg, stress, anxiety, and depression, has been identified among health-care workers in Saudi Arabia. ${ }^{12}$

To address these challenges, there is a need to deploy effective and innovative strategies that can streamline health-care operations, increase operational efficiency, lower costs and deliver high-quality health-care services. In this context, innovative eHealth technologies enhanced by suchtechniques as artificial intelligence (AI), machine learning (ML), and the Internet of things (IoT) with smart devices and wearables can improve health-care services, ${ }^{13}$ and implementing the gig economy into health care (gig eHealth) can enhance operational efficiency in health-care systems. $^{14}$ In this context, this study investigates and compares the gig economy and eHealth as prospects for future health care in Saudi Arabia.

\section{Literature Review}

\section{Gig Economy in Health Care}

According to a recent survey conducted by Deloitte, ${ }^{15}$ societal impact (diversity, equality, and environment) was the major factor used for evaluating the success of organizations, followed by customer satisfaction, employee satisfaction, financial performance, and regulatory adherence. This reflects the importance given to society and its development in every sector of business. However, such issues as economic uncertainty, job insecurity, and increasing motivation for self-employment are factors leading to the change in attitudes of individuals toward employment. $^{16}$ A majority of Generation $\mathrm{Y}$ and Generation $\mathrm{Z}$ are increasingly adopting the ideas of independent work, freedom of choice in selecting work, and flexibility of working hours to complete work requirements, reflecting an increasing trend toward selfemployment rather than full-time employment, where they are bound by organizational policies and regulations. This has led to the development of an economic model called the gig economy, in which individuals (gig workers) can freely choose gigs (tasks) provided by companies or clients on an intermediary platform (freelancing) for agreed payment terms, not as an employee of the company but as an independent worker. ${ }^{17}$ There is an increasing trend in the adoption of gig-economy models across the globe. For example, in the US the number of gig workers increased from 3.7 million in 2014 to 62.2 million in $2019 .^{18,19}$ In addition, the gross global volume of the gig economy in 2019 was identified to be US\$248.3 billion, and projected to reach $\$ 455.2$ billion by $2023 .^{20}$ Therefore, the prospects for the gig economy appear to be huge, given the flexibility and changing trends.

The gig infrastructure can be categorized into three areas of services: gig workers, clients, and intermediaries. ${ }^{21}$ Gig workers are independent workers who can post their profiles on freelancing platforms and express interest in any gigs or tasks posted on the platform by clients. Clients or companies can post various gigs on the freelancing platforms, and have the flexibility of recruiting gig workers spread across the world. Intermediary platforms are freelancing platforms where both clients and users can post their tasks and profilesand interact to enter into contracts for tasks with agreed payment and schedule terms. Intermediary platforms, such 
as Upwork, act as a bridge between clients and gig workers. ${ }^{21}$ Companies can recruit gig workers from developing countries, who come at low labor costs, and gig workers can have access to clients across the globe, which increases the opportunities for employment, good income, and flexibility and independence at work. ${ }^{22}$ As such, the gig economy can benefit both companies and gig workers.

In the context of health care, there are various services, such as on-demand diagnosis and treatment, booking appointments, and online consultations, and other health-care tasks that may include administrative and clerical work, information-system development, management, and research-related work, such as surveys and trials, which makes them appropriate for adopting a gig culture. ${ }^{14}$ For effective integration, it is necessary to identify a suitable business model and classify various types of gigs in the health-care system. In this context, a gig-economy model ${ }^{23-25}$ is refigured in the context of health care, as shown in Figure 1.

Location-based and web-based platforms are used in gig health systems. Location-based services are used for providing health-care services within a specific region and can be accessed from intermediary platforms, whereas web-based platforms are mainly used for providing services that can be managed online. ${ }^{23}$ Both location-based and web-based platforms can be further categorized by the type of gig assignment, which includes assigning gig work to an individual and assigning gig work to multiple people. For example, using freelancing platforms, such as Upwork, companies or clients can assign particular work to individuals, such as online consultation with a physician who has a profile on Upwork. Similarly, using intermediary platforms, such as Appen, companies can assign tasks to a crowd (a group of people who are registered on Appen and form a group specialized in a set of activities), which may include small tasks, such as system maintenance or data entry, and major tasks, such as systems integration of updates with predictive technologies in various areas of health care, such as diagnosis (radiology) and information management (electronic health records). ${ }^{26,27}$ Web-based and location-based gigs can be classified into various categories, such as software development and technology, creative and multimedia, clerical and data entry, sales and marketing support, and professional

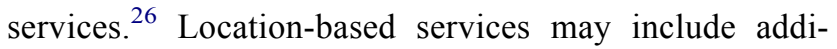
tional services, such as personal consultation, diagnosis, and treatment, where users can book appointments for personal consultation and treatment on freelancing platforms, in contrast to online consultation on web-based gig platforms.

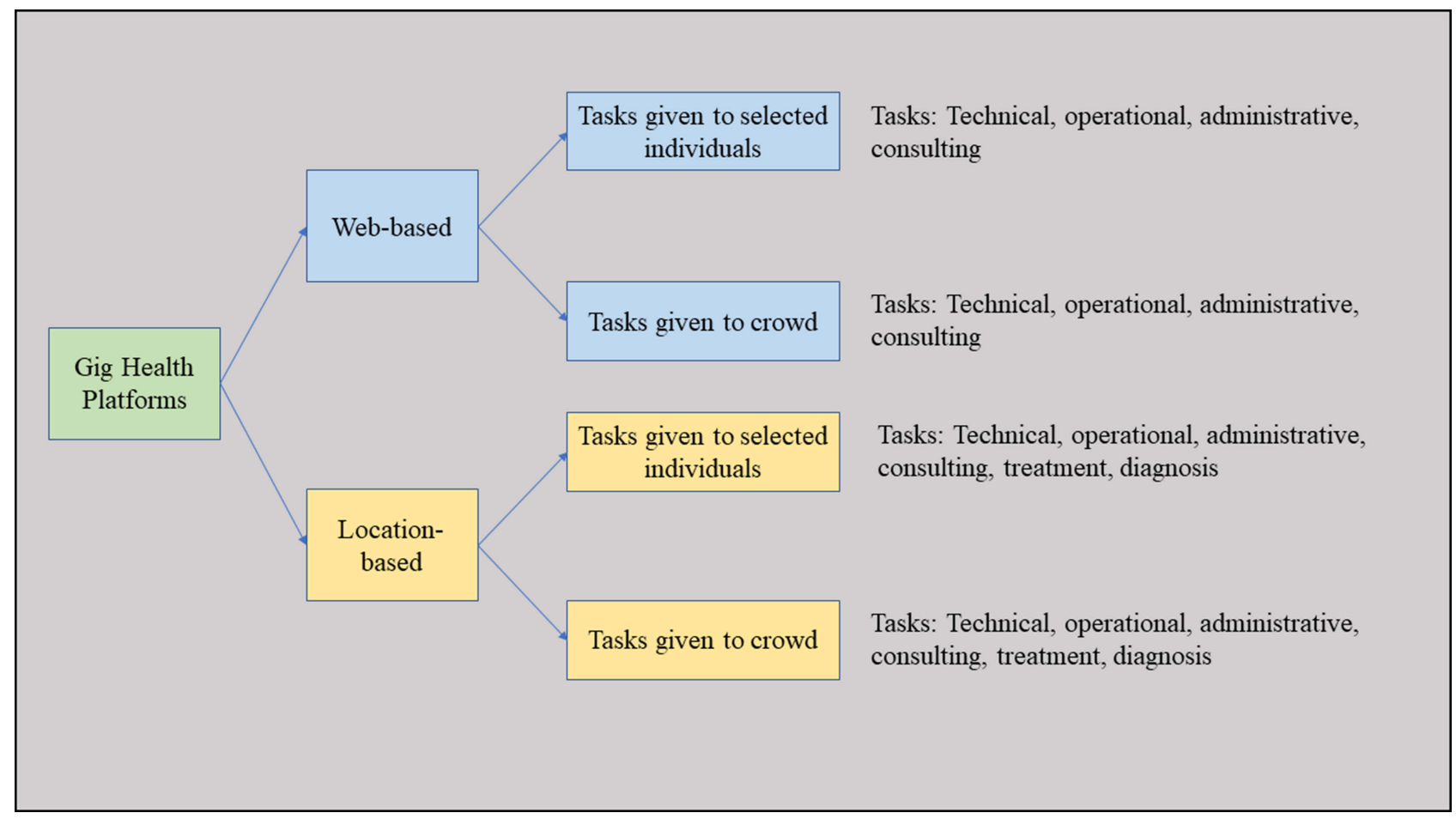

Figure I Gig-economy health-business model. 


\section{eHealth}

eHealth is an approach to providing health-care services and managing health-care operations supported by Internet and communication technologies. ${ }^{28}$ There are various definitions of eHealth, as it covers multiple dimensions of health-care services and technology applications. ${ }^{29}$ It can also be linked to services provided through such devices as mobiles and smartphones - mHealth. ${ }^{30}$ A broad definition of eHealth given by Eysenbach ${ }^{29}$ includes the concepts of health care, technology, and psychological aspects:

eHealth is an emerging field in the intersection of medical informatics, public health and business, referring to health services and information delivered or enhanced through the Internet and related technologies. In a broader sense, the term characterizes not only a technical development, but also a state-of-mind, a way of thinking, an attitude, and a commitment for networked, global thinking, to improve health care locally, regionally, and worldwide by using information and communication technology.

Accordingly, ten objectives were identified in relation to eHealth, which included increasing efficiency, enhancing quality, evidence-based interventions, empowerment, encouragement, education, enabling information exchange, extending scope of health care, ethics, and equity. ${ }^{29}$ However, for effective adoption of eHealth, these must have such features as ease of use, and entertainment (fun and engagement). eHealth applications have been identified to be effective in managing various healthcare operations, such as booking appointments, online consultations, managing critical illnesses, eg, diabetes, through self-management approaches, health monitoring, and fitness. ${ }^{31-35}$ In addition, recent technologies being developed in AI, ML, and deep-learning neural networks have led to the development of various prospects of using the IoT in eHealth approaches. IoT technologies, such as wearables, sensors, and transmitters, can be used for monitoring and diagnosing various health-related data and automating preventive treatments, feedbacks, and alert systems, which can effectively reduce interventions by medical professionals in managing various diseases and also maintaining fitness. ${ }^{36,37}$ However, challenges related to data security and privacy, eHealth literacy of health-care consumers, and integration of advanced technologies into traditional health-care systems are major factors affecting the large-scale implementation of eHealth technologies across the world. ${ }^{38}$
In Saudi Arabia, implementation of eHealth technologies has been gaining momentum, with various initiatives taken by the government to digitize health care as a part of Vision 2030. A study in $2011^{39}$ identified that there are only three of the 19 major government hospitals had adopted electronic health-record systems, while in a study conducted in $2020,{ }^{40}$ among 18 hospitals in the eastern region of Saudi Arabia, all hospitals were implementing health-information systems at various levels. In addition, it was observed that most of the functionalities of electronic clinical documentation and viewing categories were fully implemented in a majority of hospitals, about half the functions in the decision-support category were either fully implemented or not implemented, and most of the bar-coding functions were fully implemented in a majority of the hospitals, except for the supply-chain function, which was not available in $44 \%$ of the hospitals. ${ }^{40}$ The findings from the studies ${ }^{39,40}$ indicate the progress in the deployment of eHealth technologies in Saudi Arabia. However, issues related to organizational operations, cultures, end-user attitudes toward eHealth projects, the lack of specialized human resources to implement eHealth systems, ${ }^{13}$ poor eHealth frameworks, and lack of strategies managing information, creating awareness, increasing accessibility and reachability, promoting self-management and self-collaboration, promoting electronic services, and extensive stakeholder engagement ${ }^{41}$ were identified.

\section{Methods}

\section{Study Design}

Considering the purpose of this study, which was to identify the prospects of gig health and eHealth for the Saudi Arabian health-care system, we realized there was a need for in-depth analysis of opinions and perceptions of experts. Qualitative approaches, such as semistructured interviews, can be an effective approach to collect data related to participants' opinions and perceptions in research. ${ }^{42,43}$ In addition, semistructured interviews have been used in various social research studies, as they can allow one-to-one interaction with predefined questions and facilitate instant formulation of questions during the interviews, depending on the course of the discussion. ${ }^{43-}$ 45 An initial interview questionnaire in Arabic was designed with questions related to the concepts of the gig economy and eHealth approaches and techniques identified from the studies reviewed in the 
literature. ${ }^{13,15-41}$ Due to COVID-19 regulations, safety procedures were followed and interviews conducted using Zoom. Interviews were audiorecorded and later transcribed and analyzed using NVivo 12.

\section{Recruitment and Sampling}

As the study focused on prospects for the gig economy and eHealth in Saudi Arabia, an expert sample, including executives from the Ministry of Health and Ministry of Communications and Information Technology, and healthcare professionals, including physicians and administrative managers, were recruited. Requests for interviews were made via email to 26 executives, 18 physicians, and 12 administrative managers working in public hospitals, with details of the study, including its objectives and privacy policy of maintaining participants' anonymity. Responses were received from 32 participants. Due to issues in scheduling, 13 did not participate in the interviews, and so a final interview sample of 19 interviewees was achieved. Interviews were conducted over 9 weeks between November 2, 2020 and December 28, 2020.

Of the 19 interview participants, eleven were male and eight female. Five executives from the Ministry of Health, four from the Ministry of Communications and Information Technology, six physicians, and four administrative managers from public hospitals were interviewed.

\section{Data Analysis}

Audio recordings of the interviews were initially transcribed to Arabic and later translated to English using NVivo. Subsequent transcriptions were analyzed by a group of three researchers, who followed the methodological principles of thematic analysis, ${ }^{46}$ focusing on themes and patterns of behavior.

\section{Ethics}

Ethics approval for the study was received from Imam Abdulrahman Bin Faisal University. Participants were ensured of their privacy and anonymity. They were also fully informed about the purpose of the study and data usage, which was strictly for academic purposes. Participants consented to publication of anonymized responses. Accordingly, all the guidelines proposed by the university's ethics committee were followed.

\section{Results and Discussion}

The data collected from the interviews is analyzed under three themes: opportunities, challenges, and prospects.

\section{Opportunities}

Various opportunities were identified in relation to the gig economy and eHealth. A majority of the interviews referred to employment growth (eleven of 19), economic growth (13 of 19), and sustainable development (ten of 19) as the major opportunities for the gig economy. In addition, improved operational efficiency (13 of 19), speed of delivery and lower costs of health-care services (12 of 19), providing quality services (ten of 19), and effective decision-making (13 of 19) were the major opportunities identified in health care with the adoption of eHealth technologies by the participants. With respect to the gig economy in health care, one of the executives from the Ministry of Health stated that:

\begin{abstract}
The gig economy can enhance the participation of women and speciallyabled in the employment sector and increase the availability of skilled workers for the companies, enhancing flexibility in recruiting the workers. At the same time, it can support workers, especially from rural areas, who are a bit conservative, and support them in opting for tasks at their convenience by working from home, thus leading to balanced regional development and greater participation of the unused workforce. (Interviewee 15)
\end{abstract}

Different benefits were observed in relation to companies, gig workers, and society as a whole in terms of supporting growth and the economy. In accordance with other findings, ${ }^{15}$ societal impact was considered a major motivational factor for deploying the gig economy in health care. Moreover, previously identified benefits of the gig economy ${ }^{47}$ can include the dormant workforce, especially women and speciallyabled, in the economy, and empower them, acording to the interviewees. Similarly, focusing on eHealth, an executive from the Ministry of Health stated that:

eHealth has enormous advantages in improving healthcare services. We are considering the deployment of new innovative and predictive technologies for enhancing decision-making in health care, such as assessing X-ray reports, diagnosing radiology reports, etc. At the same time, we are encouraging the adoption of eHealth in rural areas by creating awareness, so that access to healthcare services can be improved and help in effective 
management of health-care services and operations. (Interviewee 8)

While the gig economy provides opportunities by increasing the availability of skilled independent workers and supporting economic growth, eHealth on the other hand improves health-care services and operations through the adoptiion of innovative technologies. A lack of skilled human resources, poor stakeholder engagement, and issues in implementation were identified as challenges in adopting eHealth technologies in Saudi Arabia, ${ }^{13,41}$ which can be effectively addressed by the gig economy and promote sustainable growth in the health-care sector.

\section{Challenges}

Various challenges were identified in relation to the gig economy by the interviewees. Most of the interviewees referred to lack of clear regulations and legal framework (13 of 19) and issues of health data security and consumer privacy (15 of 19) regarding incorporation of the gig economy into the system, while few interviewees referred to the gig economy as an unstable business model (four of 19) in the Saudi Arabian health care system, supporting other findings. ${ }^{17,48,49}$ In this context, one of the interviewees from the Ministry of Communications and Information Technology stated that:

Undoubtedly, the gig economy can offer a wide range of benefits, but it has to be strategically planned and implemented, as there are various challenges in integrating it. Especially, the issue of health consumers' privacy and security, issues in recruiting workers for short-term tasks, ensuring the compliance of health-care standards and regulations in this process, and most importantly building trust among the stakeholders can be very challenging. (Interviewee 4)

It is clear that there are many operational challenges identified in integrating the gig economy and health care, though there are benefits associated with the former. However, most of these can be managed by developing clear policies and regulations, in which eHealth can be very much useful. For example, one of the physicians from a public hospital in Riyadh stated that:

I think we are not ready yet to use the gig economy. But I believe that it can be of great potential if we can address the challenges and implement it effectively. Issues such as frequently changing staff may create issues in delivering care to the patients in hospitals. Moreover, it may lead to lack of responsibility among the gig workers employed in hospitals on a contract basis. (Interviewee 18)

Creating a sense of responsibility and liability is one of the important challenges, as gig workers are not bound by organizational regulations, which may further affect the quality of services and treatment and also health consumers' relationships with the hospitals. In a similar context, one of the administrative managers stated that:

Being overburdened with health-care tasks may affect the lifestyles and health of the gig workers. A full-time employee may be allocated fixed working hours as per the hospital rules, but a gig worker has to follow the rules as mentioned in the contract, which may sometimes lead to increased working hours and lower payments by the management to reduce the costs. (Interviewee 6)

This reflects the challenge associated with gig-worker exploitation by hospitals. With increased burdens, the health of the gig workers may be seriously affected, leading to stress and anxiety. ${ }^{50,51}$ Similarly, focusing on eHealth, one of the executives from the Ministry of Health stated that:

eHealth to a great extent can facilitate the deployment of gig culture, mostly by digitizing all health-care operations. However, the main issue is integrating gig culture into the eHealth framework, which may need various infrastructural and operational changes. (Interviewee 1)

The issue of managing the gig culture in eHealth is another important challenge observed by the interviewees. However, most of the interviewees (12 of 19) reflected the opinion that the challenges associated with the gig economy and eHealth can be addressed by using innovative technologies and strategic approaches.

\section{Prospects}

There are many prospects for the gig economy and eHealth. Hospitals can access skilled a workforce across the globe, ${ }^{22}$ which can significantly improve their competitive edge and enhance their talent resources and knowledge base. However, a few changes, such as digitization and supporting technologies, are essential. In this context, one of the interviewees from the Ministry of Communications and Information Technology stated that:

In accordance with Vision 2030, we are implementing digitization across various sectors. Health care is one of the primary sectors we are currently focusing on. Various programs have been launched to speed up the digital 
transformation process of health-care operations, especially in the areas of maintaining EHRs, new automated technologies for diagnosis, and self-management procedures. This can facilitate the implementation of the gig economy, as digitization is the basis for employing a gig economy. To further strengthen the gig approach, we have initiated various skill-development programs as a part of Saudi application. (Interviewee 3)

This statement indicated that the country is in the process of digital transformation in health care, which can be understood from other findings. ${ }^{39,40}$ The General Organization for Social Insurance, in collaboration with the Ministry of Labour and Social Development and the Human Resources Development Fund, launched various programs to support gig workers as part of Vision 2030. Training in 78 designated professional roles was provided that also included healthcare professionals, allowing youth to explore gig opportunities and a reduction in unemployment. ${ }^{52}$ Accordingly, Saudi Arabia is committed to raising women's participation in the economy, especially in health care, where demand is very high, by launching various empowerment, training, and leadership programs. ${ }^{52}$

Similarly, eHealth was also referred to by the majority of interviewees in relation to various prospects in future health care, especially supporting the elderly and critically ill patients with advanced robotic health services (eleven of 19), automated devices for health monitoring (13 of 19), and increasing automated health services (ten of 19) at clinics. In this context, one of the executives from the Ministry of Health stated that:

We are looking forward to innovative and predictive technologies in health care. Our initiatives in accordance with Vision 2030 have acknowledged the use of AI and deeplearning technologies in health care. Our newly proposed city, Neom, is an example of integrating innovative health infrastructure with the community. We are proposing innovative health-care infrastructure in the city backed by research and innovation from entrepreneurs across the world working within the city, which can also promote gig culture. (Interviewee 11)

Various eHealth plans have been proposed for the development of health-care infrastructure within the city connected through high-end technology infrastructure based on AI and predictive technologies for providing high-end medical treatment, complemented by the comprehensive wellness services offered at medical resorts. ${ }^{53,54}$ In addition, the proposal to make Neom an international hub for entrepreneurs, researchers, and innovators can enhance the integration of the gig economy as a model for a futuristic city. ${ }^{55}$ Accordingly, one of the physicians stated that:

The future in health care would be dominated by eHealth technologies in delivering innovative solutions for managing health, and also gig-work culture for making the most effective utilization of human resources in a way that best benefits both health-care organizations and health-care workers. (Interviewee 16)

This statement indicated that there are prospects for both eHealth and the gig economy in the Saudi Arabian healthcare system.

\section{Conclusion}

This study identified opportunities, challenges, and prospects for gig health and eHealth in the context of Saudi Arabia. The findings suggest that there is huge scope for implementing the gig-economy model in eHealth operations. Moreover, such initiatives as digitization and tailoring to theSaudi context as part of the Vision 2030 programme have greatly contributed to the digital transformation of health-care operations, providing an innovative path for future health-care by the adoption of innovative technologies, such as AI and ML. In addition, the digitization approach can largely favor the gig economy, as most health gigs can be managed online with less personal intervention. However, the need for a strategic approach with clear formulation of legal and regulation policies was identified for the effective integration of gig culture in to the eHealth system, indicating the prospects for the Saudi Arabian health-care system. The findings in this study can have practical implications, as they can guide health-care decision-makers in promoting and facilitating the implementation of the gig economy in the Saudi Arabian health-care system. In addition, this study addresses gaps in gig-economy and eHealth literature by presenting various challenges, opportunities, and prospects associated with integrating the gig economy and eHealth. With a lack of literature in the context of integrating the gig economy with the health-care sector in Saudi Arabia, our findings can guide future research. Assessment of the digital gig-economy ecosystem in light of the COVID-19 crisis, development of gigeconomy policies, frameworks, and platforms, and readiness of ICT infrastructure for adopting the gig economy can be considered in future studies.

\section{Disclosure}

The author reports no conflicts of interest for this work. 


\section{References}

1. PwC. Chronic diseases and conditions are on the rise [Internet]. PwC; 2021 [cited January 28, 2021]. Available from: https://www.pwc. $\mathrm{com} / \mathrm{gx} / \mathrm{en} /$ industries/healthcare/emerging-trends-pwc-healthcare /chronic-diseases.html\#: :text=According $\% 20$ to $\% 20$ the $\% 20$ World $\%$ 20 Health,most $\% 20$ significant $\% 20$ in $\% 20$ developing $\% 20$ nations. Accessed July 5, 2021.

2. World Health Organization. Health workforce [Internet]. Who.int; 2021 [cited January 22, 2021]. Available from: https://www.who.int/data/gho/ data/themes/topics/health-workforce. Accessed July 5, 2021.

3. Global Health Index. 2019 Global health security index [Internet]. ghsindex.org; 2021 [cited January 22, 2021]. Available from: https:// www.ghsindex.org/. Accessed July 5, 2021.

4. Rahman R, Al-Borie H. Strengthening the Saudi Arabian healthcare system: role of vision 2030. Int J Healthc Manag. 2020;1-9. doi:10.1080/20479700.2020.1788334

5. Ministry of Health. Vision 2030. [Internet] [cited January 22, 2021]. Available from: https://www.vision2030.gov.sa/en/node/68. Accessed July 5, 2021.

6. Omnia Health. Vision 2030 represents a structural shift in the Saudi healthcare sector [Internet] [cited January 22, 2021]. Available from: https:/insights.omnia-health.com/hospital-management/vision-2030represents-structural-shift-saudi-healthcare-sector. Accessed July 5, 2021.

7. Falatah R, Salem O. Nurse turnover in the Kingdom of Saudi Arabia: an integrative review. $J$ Nurs Manag. 2018;26(6):630-638. doi: 10.1111 jonm. 12603

8. Global Health Index. 2019 Global health security index for Saudi Arabia [Internet]. ghsindex.org; 2021 [cited January 22, 2021]. Available from: https://www.ghsindex.org/country/saudi-arabia/. Accessed July 5, 2021.

9. Robert A, Al Dawish M. The worrying trend of diabetes mellitus in Saudi Arabia: an urgent call to action. Curr Diabetes Rev. 2020;16 (3):204-210. doi:10.2174/1573399815666190531093735

10. Tyrovolas S, El Bcheraoui C, Alghnam S, et al. The burden of disease in Saudi Arabia 1990-2017: results from the Global Burden of Disease Study 2017. Lancet Planet Health. 2020;4(5):e195-e208. doi:10.1016/S2542-5196(20)30075-9

11. Global health exhibition. 2019 Saudi Arabia healthcare industry overview [Internet]. Globalhealthsaudi.com; 2021 [cited January 25, 2021]. Available from: https://www.globalhealthsaudi.com/content/dam/ Informa/globalhealthsaudi/downloads/GHE19-KSA-HEALTHCAREINDUSTRY-OVERVIEW.pdf. Accessed July 5, 2021.

12. Al-Hanawi M, Mwale M, Alshareef N, et al. Psychological distress amongst health workers and the general public during the COVID-19 pandemic in Saudi Arabia. Risk Manag Healthc Policy. 2020;13:733-742.

13. Alsulame K, Khalifa M, Househ M. eHealth in Saudi Arabia: current trends, challenges and recommendations. Stud Health Technol Inform. 2015;213:233-236. PMID: 26153002.

14. Alanezi F, Alanzi T. A gig mHealth economy framework: scoping review of internet publications. JMIR Mhealth Uhealth. 2020;8(1): e14213. PMID: 31939745; PMCID: PMC6996725. doi:10.2196/ 14213

15. Deloitte and Forbes Insights. Success personified in the fourth industrial revolution: four leadership personas for an era of change and uncertainty. Deloitte Insights. January 20, 2019.

16. Manyika J, Lund S, Bughin J, Robinson K, Mischke J, Mahajan D. Independent Work: Choice, Necessity, and the Gig Economy. McKinsey Global Institute; 2016:1-16.

17. Roy G, Shrivastava AK. Future of gig economy: opportunities and challenges. IMI Konnect. 2020;9(1):14-27.

18. Statista. Number of freelancers in the United States from 2017 to 2028(in millions) [Internet]. Available from: https://www.statista. com/statistics/921593/gigeconomy-number-of-freelancers-us/. Accessed July 5, 2021.
19. Pofeldt E. Freelance economy continues to roar [Internet]. Available from: https://www.forbes.com/sites/elainepofeldt/2018/10/31/freelan cing-economy-continues-to-roar/?sh $=7 \mathrm{c} 8 \mathrm{c} 12 \mathrm{~b} 07 \mathrm{df} 4$. Accessed July 5, 2021.

20. Statista. Gig economy: projected gross volume 2023 [Internet]. Statista; 2021 [cited January 27, 2021]. Available from: https:// www.statista.com/statistics/1034564/gig-economy-projected-grossvolume/. Accessed July 5, 2021.

21. Meijerink J, Keegan A. Conceptualizing human resource management in the gig economy. J Manag Psychol. 2019;34(4):214-232. doi:10.1108/JMP-07-2018-0277

22. Beerepoot N, Lambregts B. Competition in online job marketplaces: towardsa global labour market for outsourcing services? Global Netw. 2015;15(2):236-255. doi:10.1111/glob.12051

23. Berg J, Furrer M, Harmon E, Rani U, Silberman MX. Digital Labour Platforms and the Future of the Work. International Labor Organization (ILO) Report. 2018.

24. Pesole A, Urzí Brancati MC, Fernández-Macías E, Biagi F, González Vázquez I. Platform workers in Europe [online]; 2021. Available from: https://publications.jrc.ec.europa.eu/repository/bitstream/ JRC112157/jrc112157_pubsy_platform_workers_in_europe_science_ for policy.pdf. Accessed July 5, 2021.

25. Schmidt F. Digital Labour Markets in the Platform Economy: Mapping the Political Challenges of Crowd Workand Gig Work. Friedrich-Ebert-Stiftung; 2017. ISBN: 978-3-95861-745-2.

26. Kässi O, Lehdonvirta V. Online labour index: measuring the online gig economy for policy and research. Technol Forecast Soc Change. 2018;137(137):241-248. doi:10.1016/j.techfore.2018.07.056

27. Appen. Artificial intelligence for healthcare [Internet]. Appen; 2021 [cited January 27, 2021]. Available from: https://appen.com/indus tries/healthcare/. Accessed July 5, 2021.

28. Della Mea V. What is e-Health (2): the death of telemedicine? $J$ Med Internet Res. 2001;3(2):e22. doi:10.2196/jmir.3.2.e22

29. Eysenbach G. What is e-health? J Med Internet Res. 2001;3(2):e20. doi:10.2196/jmir.3.2.e20

30. Oh H, Rizo C, Enkin M, Jadad A, Powell J, Pagliari C. What is eHealth (3): a systematic review of published definitions. $J$ Med Internet Res. 2005;7(1):e1. doi:10.2196/jmir.7.1.e1

31. Kim H, Goldsmith JV, Sengupta S, et al. Mobile health application and e-Health literacy: opportunities and concerns for cancer patients and caregivers. J Cancer Educ. 2019;34(1):3-8. doi:10.1007/s13187017-1293-5

32. Nachabe L, Raiyee R, Falou O, Girod-Genet M, ElHassan B Diabetes Mobile Application as a Part of Semantic Multi-Agent System for E-Health, 2020. IEEE 5th Middle East and Africa Conference on Biomedical Engineering (MECBME), Amman, Jordan, 2020; 1-5, doi: 10.1109/MECBME47393.2020.9265125.

33. Torres J, Vaca C e-Health and fitness in Ecuador: a social media based analysis. 2017 Fourth International Conference on eDemocracy \& eGovernment (ICEDEG), Quito, 2017; 132-139, doi: 10.1109/ ICEDEG.2017.7962523.

34. Bellei E, Biduski D, Cechetti N, De Marchi A. Diabetes mellitus m-health applications: a systematic review of features and fundamentals. Telemed e-Health. 2018;24(11):839-852. doi: $10.1089 / \mathrm{tmj} .2017 .0230$

35. Alanzi T. A review of mobile applications available in the app and google play stores used during the COVID-19 outbreak. J Multidiscip Healthc. 2021;14:45-57. doi:10.2147/JMDH.S285014

36. Kodali RK, Swamy G, Lakshmi B An implementation of IoT for healthcare. 2015 IEEE Recent Advances in Intelligent Computational Systems (RAICS), Trivandrum, 2015; 411-416, doi: 10.1109/ RAICS.2015.7488451.

37. Elhoseny G, Ramírez-González G, Abu-Elnasr M, Shawkat SA, Arunkumar N, Farouk A. Secure medical data transmission model for IoT-based healthcare systems. IEEE Access. 2018;6:20596-20608. doi:10.1109/ACCESS.2018.2817615 
38. Selvaraj S, Sundaravaradhan S. Challenges and opportunities in IoT healthcare systems: a systematic review. SN Appl Sci. 2020;2(1):139. doi:10.1007/s42452-019-1925-y

39. Sulaiman B, Alharti H, Mahalli AAE, Jabali A, Al-Qahatani M, A1kahtani N. Annual survey on the level and extent of usage of electronic health records in government-related hospitals in eastern province, Saudi Arabia. Perspectives in health information management [Internet]; 2021. Available from: https://perspectives. ahima.org/annual-survey-on-the-level-and-extent-of-usage-ofelectronic-health-records-in-government-related-hospitals-in-easternprovince-saudi-arabia/. Accessed July 5, 2021.

40. Alsalman D, Alumran A, Alrayes S, et al. Implementation status of health information systems in hospitals in the eastern province of Saudi Arabia. Inform Med Unlocked. 2021;22:100499. doi:10.1016/j. imu.2020.100499

41. Alsharif A. Applying eHealth for pandemic management in Saudi Arabia in the context of COVID-19. JMIR Med Inform. 2020;8(11): e19524. doi:10.2196/19524

42. Smith JA. Semi structured interviewing and qualitative analysis. In: Smith JA, Harre R, Van Langenhove L, editors. Rethinking Methods in Psychology. Sage Publications; 1995:9-26.

43. DeJonckheere M, Vaughn LM. Semistructured interviewing in primary care research: a balance of relationship and rigour. Fam Med Community Health. 2019;7(2):e000057. doi:10.1136/fmch-2018000057

44. Flick U, Flick U. The Sage Handbook of Qualitative Data Collection. SAGE Publications Ltd; 2017.

45. Evans C, Lewis J. Analysing Semi-Structured Interviews Using Thematic Analysis: Exploring Voluntary Civic Participation Among Adults. 2018.

46. Aronson J. A pragmatic view of thematic analysis. Qual Rep. 1994;2 (1):1-3.
47. Harpur P, Blanck P. Gig workers with disabilities: opportunities, challenges, and regulatory response. J Occup Rehabil. 2020;30 (4):511-520. doi:10.1007/s10926-020-09937-4

48. Barzilay AR, Ben-David A. Platform inequality: gender in the gig-economy. Seton Hall Law Rev. 2016;47:393-431.

49. Horney N. The gig economy: a disruptor requiring HR agility. People Strat. 2016;39(3):20-27.

50. Bajwa U, Gastaldo D, Di Ruggiero E, et al. The health of workers in the global gig economy. Global Health. 2018;4(1):124. doi:10.1186/ s12992-018-0444-8

51. Gaskell A. The mental health challenges of the gig economy [Internet]. Forbes; 2021 [cited January 25, 2021]. Available from: https://www.forbes.com/sites/adigaskell/2020/03/10/the-mentalhealth-challenges-of-the-gig-economy/?sh=1cc1 fad33020. Accessed July 5, 2021.

52. Deloitte. Leading the social enterprise: reinvent with a human focus [Internet]. Www2.deloitte.com; 2021 [cited January 28, 2021]. Available from: https://www2.deloitte.com/content/dam/Deloitte/xe/ Documents/human-capital/ME-HC-Trends-2019.pdf. Accessed July $5,2021$.

53. Zawya. Saudi's NEOM smart city to become first 'health tech' capital of the world [Internet] [cited January 22, 2021]. Available from: https://www.gitex.com/breaking-news/saudis-neom-smart-city-tobecome-first-health-tech-capital-of-the-world. Accessed July 5, 2021.

54. Molina RM. Neom could become the first 'health tech' capital of the world [Internet] [cited January 22, 2021]. Available from: https:// www.arabnews.com/node/1707136/neom-could-become-first- $\%$ E2\% $80 \% 98$ health-tech $\%$ E2\%80\%99-capital-world. Accessed July 5, 2021.

55. NEOM. NEOM - it's time to draw the line [internet]. Neom.com; 2021 [cited January 15, 2021]. Available from: https://www.neom. com/whatistheline/. Accessed July 5, 2021.
Journal of Multidisciplinary Healthcare

\section{Publish your work in this journal}

The Journal of Multidisciplinary Healthcare is an international, peerreviewed open-access journal that aims to represent and publish research in healthcare areas delivered by practitioners of different disciplines. This includes studies and reviews conducted by multidisciplinary teams as well as research which evaluates the results or conduct of such teams or healthcare processes in general. The journal covers a very wide range of areas and welcomes submissions from practitioners at all levels, from all over the world. The manuscript management system is completely online and includes a very quick and fair peer-review system. Visit http://www.dovepress.com/testimonials. php to read real quotes from published authors. 